\title{
Integrated random processes exhibiting long tails, finite moments, and power-law spectra
}

\author{
Jaume Masoliver* and Miquel Montero \\ Departament de Física Fondamental, Universitat de Barcelona, Diagonal 647, E-08028 Barcelona, Spain \\ Alan McKane \\ Department of Theoretical Physics, University of Manchester, Manchester M13 9PL, United Kingdom \\ (Received 28 July 2000; revised manuscript received 14 December 2000; published 25 June 2001)
}

\begin{abstract}
A dynamical model based on a continuous addition of colored shot noises is presented. The resulting process is colored and non-Gaussian. A general expression for the characteristic function of the process is obtained, which, after a scaling assumption, takes on a form that is the basis of the results derived in the rest of the paper. One of these is an expansion for the cumulants, which are all finite, subject to mild conditions on the functions defining the process. This is in contrast with the Lévy distribution-which can be obtained from our model in certain limits-which has no finite moments. The evaluation of the spectral density and the form of the probability density function in the tails of the distribution shows that the model exhibits a power-law spectrum and long tails in a natural way. A careful analysis of the characteristic function shows that it may be separated into a part representing a Lévy process together with another part representing the deviation of our model from the Lévy process. This allows our process to be viewed as a generalization of the Lévy process that has finite moments.
\end{abstract}

DOI: 10.1103/PhysRevE.64.011110

PACS number(s): 05.40.-a, 89.90.+n, 87.23.Ge

\section{INTRODUCTION}

The nature of the probability distribution of stock market prices has been discussed quantitatively for over a century [1]. An early conjecture that the distribution was Gaussian was found not to be a good fit, largely because of the long tails found in financial data [2]. A later suggestion that the Lévy distribution was a better fit seemed more promising, since this distribution does at least have long tails [3]. On the other hand, this distribution has no finite moments, which is a severe limitation. The solution of truncating the distribution in order to obtain finite moments is rather ad hoc and artificial [4]. Moreover, the Lévy distribution has "too fat tails"- as opposed to the "too thin tails" of the Gaussian - to give a good fit to data [5].

This paper explores in more detail a model previously introduced [6] in order to resolve these difficulties and gives a more complete explanation of the appearance of nonGaussian and self-similar fat tails in the probability distribution [5], while still keeping the important feature that all moments are finite. The model has the Lévy distribution as a limiting form and has the form of a type of "Edgeworth expansion" for the Lévy distribution. We will give the explicit form of this relationship later in the paper (the Edgeworth series is an expansion procedure that gives corrections to the Gaussian distribution in those cases where the central limit theorem applies [7]).

While the motivation for the model was the explanation of stock market data, in this paper we want to stress the more general aspects of the model, which we expect will have applications in other areas including physics. In the last few years broad distributions have been found in many areas of physics. By broad, we mean that they have fat tails—as de-

*Corresponding author. Email address: jaume@ffn.ub.es scribed above-which frequently imply that the cumulants of the distribution are not defined. In this way the Lévy distribution enters many areas of the subject [8]. For example, Lévy statistics completely characterize the properties of the spectral lines of single molecules embedded in a glass [9]. From this it is possible to deduce various low temperature properties of the glass. Lévy statistics appear in several areas in the field of quantum optics. In subrecoil laser cooling neutral atoms and ions are cooled to ultralow temperatures, so that their momentum distributions are as narrow as possible. These may be analyzed using models based on Lévy statistics $[10,11]$. Other applications in this field are to anomalous diffusion seen in optical lattices [12], the anomalous dynamics of a single ion in an optical lattice [13], and the use of Lévy statistics to optimize subrecoil Raman cooling [14]. Many experimental investigations focus on anomalous diffusion, as in an early study of diffusion in a system of micelles [15]. However, anomalous diffusion is also seen in numerous studies of dynamical systems [16-18]. These examples illustrate the ubiquity of broad distributions in physics. We hope that the ideas presented in this paper, as regards both the nature of the distribution we derive and the physical mechanism generating it, will have applications to some of the phenomena involving broad distributions that we have mentioned above or to the many others that we did not discuss.

The paper is organized as follows. In Sec. II we present the main results of the paper for clarity, since many details are quite technical. In Sec. III we describe our integrated process in detail. In Sec. IV we impose the self-scaling property on the probability distribution and obtain several relevant functions. Section V is devoted to moments and cumulants and the evaluation of the power spectrum. In Sec. VI we study the asymptotic behavior of the distribution while in Sec. VII we present, starting from our distribution, an Edgeworth-type series for the Lévy distribution. Conclusions 
are drawn in Sec. VIII and some more technical details are in the Appendices.

\section{MAIN RESULTS}

In this section we wish to state the main results of the paper without giving the derivations or being careful to state the range of validity for which they hold. We hope that this will give a good indication of the scope and nature of our results; the interested reader can then fill out this basic framework by proceeding to later sections.

As explained in the next section, our integrated process $X(t)$ is a continuous superposition of colored shot noises parametrized by $u$. It takes the form

$$
X(t)=\int_{-\infty}^{\infty}\left[\sum_{k=1}^{\infty} A_{k}(u) \phi\left(t-T_{k}(u) ; u\right)\right] d u .
$$

There are several assumptions. (i) The pulse shape function $\phi(t, u)$ is causal, i.e., $\phi(t, u)=0$ for $t<0$. (ii) The jump amplitudes and jump times, $A_{k}(u)$ and $T_{k}(u)$, are independent and identically distributed random variables with probability density functions given by $h(x, u)$ and $\psi(t, u)$, respectively, i.e., $\quad h(x, u) d x=\operatorname{Prob}\left\{x<A_{k}(u)<x+d x\right\}, \quad$ and $\psi(t, u) d t=\operatorname{Prob}\left\{t<T_{k}(u)<t+d t\right\}$. (iii) The jump times are assumed to follow a Poisson process with parameter $\lambda(u)$. (iv) Functions $\phi(t, u)$ and $h(x, u)$ satisfy the scaling forms

$$
\phi(t, u)=\phi(\lambda(u) t) \quad \text { and } \quad h(x, u)=\frac{1}{\sigma(u)} h\left(\frac{x}{\sigma(u)}\right),
$$

where $\sigma(u)$ is the standard deviation of the jump amplitudes. The model is thus specified by the four functions of a single variable $\phi, h, \sigma$, and $\lambda$. (v) The characteristic function (CF) $\tilde{p}(\omega, t)=\langle\exp [i \omega X(t)]\rangle$, which is the Fourier transform of the density $p(x, t)$, obeys the self-scaling property

$$
\tilde{p}(\omega, t)=f\left(\omega t^{1 / \alpha}\right),
$$

where $f(\xi)$ is any non-negative definite function such that $f(0)=1$ [19]. One of the main goals of this paper is to find the probability distribution of the process $X(t)$. By assuming the scaling forms given above the one-time distribution of $X(t)$ is explicitly given by the $\mathrm{CF}$

$$
\begin{aligned}
\tilde{p}(\omega, t)= & \exp \left\{-b t \omega^{\alpha} \int_{0}^{\infty} \frac{d z}{z^{1+\alpha}}\right. \\
& \left.\times\left[1-\int_{0}^{1} \tilde{h}\left(z \phi\left(\frac{b t \omega^{\alpha} s}{z^{\alpha}}\right)\right) d s\right]\right\},
\end{aligned}
$$

where $b>0$. When $\phi(x)=\theta(x)$ is the Heaviside step function then the input shot noises are white and $X(t)$ is the Lévy process

$$
\tilde{p}(\omega, t)=e^{-M t \omega^{\alpha}},
$$

where $M=b \int_{0}^{\infty} d z[1-\widetilde{h}(z)] / z^{1+\alpha}$. This provides us with an alternative interpretation of the Lévy process since, in our case, Lévy processes are continuous superpositions of families of white Poissonian shot noises.

Contrary to Lévy processes, our integrated process can have finite moments of any order; thus cumulants are given by

$$
\left\langle\left\langle X^{n}(t)\right\rangle\right\rangle=\frac{i^{-n} \widetilde{h}^{(n)}(0)}{n}(b t)^{n / \alpha} \int_{0}^{\infty} \frac{\phi^{n}(x)}{x^{n / \alpha}} d x
$$

$(n=1,2,3, \ldots)$. Note that the second cumulant (i.e., the variance), is proportional to $(b t)^{2 / \alpha}$ and $X(t)$ presents anomalous diffusion behavior [see Sec. V for a detailed discussion on limiting values and bounds for the exponent $\alpha$ and other parameters related to the asymptotic behavior of the pulse shape function $\phi(x)]$.

We define the stationary correlation function by $C(\tau)$ $=\lim _{t \rightarrow \infty}\langle X(t+\tau) X(t)\rangle$. For our process this reads

$$
C(\tau)=b \int_{0}^{\infty} \frac{d z}{z^{1+\alpha}} \int_{0}^{\infty} \phi\left(b z^{-\alpha} t^{\prime}\right) \phi\left(b z^{-\alpha}\left(t^{\prime}+\tau\right)\right) d t^{\prime} .
$$

The power spectral density of the process $X(t)$, given by the Fourier transform of the stationary correlation, is

$$
\widetilde{C}(\omega)=\frac{K}{\omega^{1+2 / \alpha}},
$$

and $X(t)$ possesses a power-law spectrum with exponent $\nu$ $=1+2 / \alpha$.

We can also perform the asymptotic analysis of the probability distribution of $X(t)$ without having to specify any particular form for $h(x)$ and $\phi(x)$, thus keeping the maximum level of generality. Specifically, we show in Sec. VI that the center of the distribution is approached by a Lévy distribution

$$
\tilde{p}(\omega, t) \approx e^{-L(t) \omega^{\delta}}, \quad \omega \rightarrow \infty,
$$

where $0<\delta<2$. We refer the reader to Sec. VI for more details and for the behavior of the tails of the distribution, which is mainly determined by the behavior of the jump probability density function (PDF) $h(x)$.

The relation to the Lévy distribution is explored in more detail in Sec. VII, where we present an alternative (and exact) expression for the $\mathrm{CF}$ which decomposes the distribution of the integrated process $X(t)$ into that of Lévy plus an additional term:

$$
\begin{aligned}
\ln \tilde{p}(\omega, t)= & \ln \tilde{p}_{\text {Lévy }}(\omega, t)-\frac{1}{\alpha} \int_{0}^{\infty}\left[\widetilde{h}\left([b t / x]^{1 / \alpha} \omega\right)\right. \\
& \left.-\widetilde{h}\left([b t / x]^{1 / \alpha} \omega \phi(x)\right)\right] d x+\int_{0}^{\infty} x \frac{\phi^{\prime}(x)}{\phi(x)} \\
& \times\left[1-\widetilde{h}\left([b t / x]^{1 / \alpha} \omega \phi(x)\right)\right] d x .
\end{aligned}
$$

Note that when $\phi(x)$ is the rectangular step function this equation reduces to the Lévy distribution. Therefore, when $\phi(x)$ is a steplike function close to the Heaviside function, 
this alternative expression can be used as the starting point of an Edgeworth-type expansion procedure giving corrections to the Lévy distribution.

\section{THE INTEGRATED PROCESS}

Let $X(t)$ be a random process formed by a continuous superposition of independent shot-noise processes

$$
X(t)=\int_{-\infty}^{\infty} Y(u, t) d u,
$$

where, for any fixed time $t, Y(u, t)$ are independent random variables for different values of the parameter $u$ [see Eq. (10) below] and for any fixed value of $u, Y(u, t)$ is a colored shot-noise process represented by a countable superposition of pulses of identical shape,

$$
Y(u, t)=\sum_{k=1}^{\infty} A_{k}(u) \phi\left(t-T_{k}(u) ; u\right),
$$

where $T_{k}(u)$ marks the onset of the $k$ th pulse, and $A_{k}(u)$ is its amplitude. Both $T_{k}(u)$ and $A_{k}(u)$ are independent and identically distributed random variables with probability density functions given by $h(a, u)$ and $\psi(t, u)$, respectively. The pulse shape $\phi(t, u)$ has to fulfill the "causality condition," i.e., $\phi(t, u)=0$ for $t<0$ [20].

We assume that the occurrence of jumps is a Poisson process, in this case the shot noise $Y(t, u)$ is Markovian, and the PDF for the time interval between jumps, $\psi(t, u) d t$ $=\operatorname{Prob}\left\{t<T_{k}(u)-T_{k-1}(u)<t+d t\right\}$, is exponential:

$$
\psi(t, u)=\lambda(u) e^{-\lambda(u) t} \quad(t \geqslant 0),
$$

where $\lambda(u)$ is the mean jump frequency, i.e., $1 / \lambda(u)$ is the mean time between two consecutive jumps [23]. We recall that jump amplitudes $A_{k}(u)$ are identically distributed (for all $k=1,2,3, \ldots$ ) and independent random variables (for all $k$ and $u$ ). In what follows we will assume that they have zero mean and a PDF $h(x, u) d x=\operatorname{Prob}\left\{x<A_{k}(u)<x+d x\right\}$ depending on a single "dimensional" parameter which, without loss of generality, we assume to be the standard deviation of jumps $\sigma(u)=\sqrt{\left\langle A_{k}^{2}(u)\right\rangle}$. That is,

$$
h(x, u)=\frac{1}{\sigma(u)} h\left(\frac{x}{\sigma(u)}\right) .
$$

Before proceeding further with the probability distribution of the integrated process $X(t)$ given by Eq. (1), we note that following Rice's method [20] one can easily obtain all the probability distributions of the shot noise $Y(t, u)$ via their CF's

$$
\tilde{p}_{Y}\left(\omega_{1}, t_{1} ; \ldots ; \omega_{n}, t_{n} ; u\right)=\left\langle\exp \left[i \sum_{k=1}^{n} \omega_{k} Y\left(t_{k}, u\right)\right]\right\rangle .
$$

In Appendix A we show that

$$
\ln \tilde{p}_{Y}(\omega, t ; u)=-\lambda(u)\left[t-\int_{0}^{t} \widetilde{h}\left(\omega \sigma(u) \phi\left(t^{\prime}, u\right)\right) d t^{\prime}\right],
$$

and (supposing that $t_{2}>t_{1}$ )

$$
\begin{aligned}
\ln \tilde{p}_{Y}\left(\omega_{1}, t_{1} ; \omega_{2}, t_{2} ; u\right)= & -\lambda(u)\left[t_{2}-\int_{0}^{t_{1}} \widetilde{h}\left(\omega_{1} \sigma(u) \phi\left(t^{\prime}, u\right)\right.\right. \\
& \left.+\omega_{2} \sigma(u) \phi\left(t^{\prime}+t_{2}-t_{1}, u\right)\right) d t^{\prime} \\
& \left.-\int_{0}^{t_{2}-t_{1}} \widetilde{h}\left(\omega_{2} \sigma(u) \phi\left(t^{\prime}, u\right)\right) d t^{\prime}\right],
\end{aligned}
$$

where $\widetilde{h}(\omega)$ is the Fourier transform of the jump PDF $h(x)$.

Let us now evaluate the probability distribution of the integrated process $X(t)$. In terms of the cumulants $\langle\langle Y(t, u)\rangle\rangle$ of the shot noise $Y(t, u)$ we see that the one-time characteristic function of $X(t)$ can be written as

$$
\tilde{p}_{X}(\omega, t)=\exp \left\{\sum_{k=1}^{\infty} \frac{(i \omega)^{k}}{k !}\left\langle\left\langle\left[\int_{-\infty}^{\infty} Y(u, t) d u\right]^{k}\right)\right\rangle\right\} .
$$

That is,

$$
\begin{aligned}
\ln \tilde{p}_{X}(\omega, t)= & \sum_{k=1}^{\infty} \frac{(i \omega)^{k}}{k !} \int_{-\infty}^{\infty} \cdots \int_{-\infty}^{\infty}\left\langle\left\langle Y\left(u_{1}, t\right) \cdots Y\left(u_{k}, t\right)\right\rangle\right\rangle \\
& \times d u_{1} \cdots d u_{k} .
\end{aligned}
$$

But by our assumptions on the process $Y(u, t)$ we have

$$
\begin{aligned}
& \left\langle\left\langle Y\left(u_{1}, t\right) \cdots Y\left(u_{k}, t\right)\right\rangle\right\rangle \\
& \quad=\left\langle\left\langle Y^{k}\left(u_{1}, t\right)\right\rangle\right\rangle \delta\left(u_{1}-u_{2}\right) \cdots \delta\left(u_{k-1}-u_{k}\right) .
\end{aligned}
$$

Therefore,

$$
\ln \tilde{p}_{X}(\omega, t)=\sum_{k=1}^{\infty} \frac{(i \omega)^{k}}{k !} \int_{-\infty}^{\infty}\left\langle\left\langle Y^{k}(u, t)\right\rangle\right\rangle d u,
$$

that is,

$$
\ln \tilde{p}_{X}(\omega, t)=\int_{-\infty}^{\infty} \ln \tilde{p}_{Y}(\omega, t ; u) d u .
$$

Note that this line of reasoning can be easily extended to the $n$ th-time distribution, with the result

$$
\begin{aligned}
& \ln \tilde{p}_{X}\left(\omega_{1}, t_{1} ; \ldots ; \omega_{n}, t_{n}\right) \\
& \quad=\int_{-\infty}^{\infty} \ln \tilde{p}_{Y}\left(\omega_{1}, t_{1} ; \ldots ; \omega_{n}, t_{n} ; u\right) d u .
\end{aligned}
$$

Going back to our integrated process we have from Eqs. $(6),(7)$ and (11),(12) that the one-time characteristic function of $X(t)$ reads 


$$
\begin{aligned}
\ln \tilde{p}(\omega, t)= & -\int_{-\infty}^{\infty} d u \lambda(u) \\
& \times\left[t-\int_{0}^{t} \tilde{h}\left(\omega \sigma(u) \phi\left(t^{\prime}, u\right)\right) d t^{\prime}\right],
\end{aligned}
$$

while the two-time $\mathrm{CF}$ is $\left(t_{2}>t_{1}\right)$

$$
\begin{aligned}
\ln \tilde{p}\left(\omega_{1}, t_{1} ; \omega_{2}, t_{2}\right)= & \int_{-\infty}^{\infty} d u \lambda(u) \\
& \times\left\{\int _ { 0 } ^ { t _ { 1 } } d t ^ { \prime } \left[\widetilde { h } \left(\omega_{1} \sigma(u) \phi\left(t^{\prime}, u\right)\right.\right.\right. \\
& \left.\left.+\omega_{2} \sigma(u) \phi\left(t^{\prime}+t_{2}-t_{1}, u\right)\right)-1\right] \\
& \left.+\int_{0}^{t_{2}-t_{1}} d t^{\prime}\left[\tilde{h}\left(\omega_{2} \sigma(u) \phi\left(t^{\prime}, u\right)\right)-1\right]\right\},
\end{aligned}
$$

where we have dropped the subscript $X$. Obviously these are formal expressions, as long as we do not provide the functional dependence of $\lambda(u)$ and $\sigma(u)$ on the parameter $u$. We will do so in the next section using scaling arguments.

We finally note that when there is only one source of noise (instead of a continuous superposition of them) then $\lambda(u)=\lambda \delta(u)$ and Eq. (13) reads

$$
\tilde{p}(\omega, t)=\exp \left\{-\lambda\left[t-\int_{0}^{t} \tilde{h}\left(\omega \sigma \phi\left(t^{\prime}\right)\right) d t^{\prime}\right]\right\}
$$

where $\sigma \equiv \sigma(0)$ and $\phi(t) \equiv \phi(t, 0)$. We thus recover the characteristic function for non-white Poissonian shot noise [21]. Equation (15) is the starting point of Ref. [22] for the analysis of fractal shot noise when $\phi(t) \propto t^{-1 / \beta}$.

\section{SCALING}

In order to proceed further we need to specify the functional forms of $\lambda(u)$ and $\sigma(u)$. Of course that form will depend on the specific features of the problem at hand. At this point we choose what seems to us one of the most general ways of proceeding; we suppose that our integrated process $X(t)$ possesses self-scaling properties. Following this path we must first assume that the pulse function is of the form

$$
\phi(u, t)=\phi(\lambda(u) t)
$$

which turns $\phi(u, t)$ into a function of the single dimensionless variable $\lambda(u) t$. Substituting this into Eq. (13), defining new integration variables $s=t^{\prime} / t$ and $z=\omega \sigma(u)$, and supposing that $\sigma(-\infty)=0$ and $\sigma(\infty)=\infty$, we obtain

$$
\ln \tilde{p}(\omega, t)=-\int_{0}^{\infty} d z \frac{\lambda t}{\omega \sigma^{\prime}}\left\{1-\int_{0}^{1} \tilde{h}(z \phi(\lambda s t)) d s\right\},
$$

where the prime on $\sigma$ denotes the derivative. We now impose the self-scaling property on the $\mathrm{CF}$, that is, we assume that $\tilde{p}(\omega, t)$ is a function of the single variable $\omega t^{1 / \alpha}$,

$$
\tilde{p}(\omega, t)=f\left(\omega t^{1 / \alpha}\right),
$$

where the arbitrary function $f(\xi)$ has to be a characteristic function, i.e., $f(\xi)$ is a non-negative definite function such that $f(0)=1$ [19]. Note that Eq. (18) implies that the shape of $p(x, t)$ is invariant under transformations in which $x t^{-1 / \alpha}$ remains constant. We will see later on that an important consequence of this is that all moments have the property $\left\langle X^{n}(t)\right\rangle \propto t^{n / \alpha}$.

On the other hand, we note that in Eq. (17) the quantities $\sigma^{\prime}$ and $\lambda$ are functions of $z$ and $\omega$. Then scaling (18) implies

$$
\lambda=B(z) \omega^{\alpha}, \quad \frac{\lambda}{\omega \sigma^{\prime}}=A(z) \omega^{\alpha},
$$

where $A(z)$ and $B(z)$ are arbitrary functions to be determined. From these two relations we get $\sigma^{\prime}=C(z) / \omega$, where $C(z)=B(z) / A(z)$. In the original variable $u$ we have

$$
\sigma^{\prime}=\frac{C(\omega \sigma(u))}{\omega}
$$

But $\sigma^{\prime}=\sigma^{\prime}(u)$ is independent of $\omega$. Therefore, the unknown function $C$ has to be of the form $C(\omega \sigma)=k \omega \sigma$ where $k$ is a constant. Hence $\sigma^{\prime}(u)=k \sigma(u)$, whence $\sigma(u)=\sigma_{0} e^{k u}$. Finally, absorbing the constant $k$ inside the variable $u$ we obtain the functional dependence of the jump variance $\sigma$ on parameter $u$,

$$
\sigma(u)=\sigma_{0} e^{u}
$$

where $\sigma_{0}$ is a constant. Moreover, we see from Eq. (19) that $\lambda=B(\omega \sigma(u)) \omega^{\alpha}$. But again $\lambda=\lambda(u)$ is independent of $\omega$. In consequence, $B(\omega \sigma)=b(\omega \sigma)^{-\alpha}$ where $b$ is an arbitrary constant. Substituting this into the first relation of Eq. (19) yields the "dispersion relation" between the mean frequency $\lambda$ and the jump variance $\sigma$ :

$$
\lambda=b / \sigma^{\alpha}
$$

The functional dependence of $\lambda$ on $u$ is obtained by combining Eqs. (21) and (22),

$$
\lambda(u)=\lambda_{0} e^{-\alpha u},
$$

where $\lambda_{0}=b / \sigma_{0}^{\alpha}$, or equivalently

$$
b=\lambda_{0} \sigma_{0}^{\alpha}
$$

Collecting results we see from Eq. (17) and Eqs. (21)-(24) that the one-time characteristic function reads 


$$
\begin{aligned}
\tilde{p}(\omega, t)= & \exp \left\{-b t \omega^{\alpha} \int_{0}^{\infty} \frac{d z}{z^{1+\alpha}}\right. \\
& \left.\times\left[1-\int_{0}^{1} \tilde{h}\left(z \phi\left(b t \omega^{\alpha} s / z^{\alpha}\right)\right) d s\right]\right\} .
\end{aligned}
$$

It is sometimes convenient to rewrite this equation and use the alternative form of $\tilde{p}(\omega, t)$ given by

$$
\tilde{p}(\omega, t)=\exp \left\{-b t \int_{0}^{\infty} \frac{d z}{z^{1+\alpha}}\left[1-\int_{0}^{1} \tilde{h}\left(z \omega \phi\left(b t s / z^{\alpha}\right)\right) d s\right]\right\},
$$

or equivalently

$$
\tilde{p}(\omega, t)=\exp \left\{b \int_{0}^{\infty} \frac{d z}{z^{1+\alpha}} \int_{0}^{t} d t^{\prime}\left[\tilde{h}\left(z \omega \phi\left(b t^{\prime} / z^{\alpha}\right)\right)-1\right]\right\} .
$$

Starting from Eq. (14) and following an analogous reasoning based on the scaling assumption, we obtain the following expression for the two-time characteristic function of the integrated process (with $t_{2}>t_{1}$ ):

$$
\begin{aligned}
\ln \tilde{p}\left(\omega_{1}, t_{1} ; \omega_{2}, t_{2}\right)= & b \int_{0}^{\infty} \frac{d z}{z^{1+\alpha}}\left\{\int _ { 0 } ^ { t _ { 1 } } d t ^ { \prime } \left[\widetilde { h } \left(z \omega_{1} \phi\left(b z^{-\alpha} t^{\prime}\right)\right.\right.\right. \\
& \left.\left.+z \omega_{2} \phi\left(b z^{-\alpha}\left(t^{\prime}+t_{2}-t_{1}\right)\right)\right)-1\right] \\
& \left.+\int_{0}^{t_{2}-t_{1}} d t^{\prime}\left[\widetilde{h}\left(z \omega_{2} \phi\left(b z^{-\alpha} t^{\prime}\right)\right)-1\right]\right\} .
\end{aligned}
$$

Equations (25)-(28) are some of the key results of the paper, since, as we will see next, they constitute a generalization of the Lévy distribution with finite moments.

\section{MOMENTS, CUMULANTS, AND POWER SPECTRUM}

We first note from Eq. (25) that if the pulse shape function is the Heaviside step function

$$
\phi(t)= \begin{cases}1 & \text { if } t>0 \\ 0 & \text { otherwise }\end{cases}
$$

then the integrated process $X(t)$ is identically a Lévy process, regardless the jump PDF $h(x)$,

$$
\tilde{p}(\omega, t)=\exp \left[-M t \omega^{\alpha}\right]
$$

where

$$
M=b \int_{0}^{\infty} \frac{d z}{z^{1+\alpha}}[1-\tilde{h}(z)]
$$

Therefore, following our model, Lévy processes can be viewed as a continuous superposition of families of rectan- gular pulses occurring at random Poisson times. The range of the exponent $\alpha$ in Lévy flights is $0<\alpha<2$. In such a case $X(t)$ has no finite moment but the first one [8]. In actual situations, one is unlikely to meet with perfect rectangular pulses (showing sudden changes); in such a case all moments can be finite and are easily evaluated from the derivatives of the characteristic function (26). Thus, for instance, the second moment is given by [note that due to Eq. (4) $\widetilde{h}^{\prime \prime}(0)=$ $-1]$

$$
\left\langle X^{2}(t)\right\rangle=b t \int_{0}^{\infty} \frac{d z}{z^{\alpha-1}} \int_{0}^{1} \phi^{2}\left(b t s / z^{\alpha}\right) d s .
$$

As an illustrative example suppose that our pulse function has the form

$$
\phi(t)= \begin{cases}1-e^{-k t} & \text { if } t>0 \\ 0 & \text { otherwise }\end{cases}
$$

where $k>0$ is a constant [note that if $k$ is large then $\phi(t)$ approaches the rectangular pulse (29)]. In Appendix B we show that [see also Eq. (42) below]

$$
\left\langle X^{2}(t)\right\rangle=D t^{2 / \alpha} \quad(2>\alpha>2 / 3),
$$

where $\quad D=\alpha b^{2} k^{-1+2 / \alpha}\left(1-2^{2 / \alpha-2}\right) \Gamma(2-2 / \alpha) /(2-\alpha)$. Since $1<(2 / \alpha)<3$, Eq. (34) clearly shows a superdiffusive behavior.

In fact, we can easily obtain a closed expression not for moments but for cumulants defined as derivatives of $\ln \tilde{p}(\omega, t)$. From Eq. (26) we have

$$
\left\langle\left\langle X^{n}(t)\right\rangle\right\rangle=i^{-n} b t \widetilde{h}^{(n)}(0) \int_{0}^{\infty} z^{n-1-\alpha} d z \int_{0}^{1} \phi^{n}\left(b t s / z^{\alpha}\right) d s .
$$

If in the double integral on the right hand side of this equation we define a new integration variable $x$ by $s=\left(z^{\alpha} / b t\right) x$ and exchange the order of integration, we get

$$
\begin{aligned}
& \int_{0}^{\infty} z^{n-1-\alpha} d z \int_{0}^{1} \phi^{n}\left(b t s / z^{\alpha}\right) d s \\
& \quad=\frac{1}{b t} \int_{0}^{\infty} \phi^{n}(x) d x \int_{0}^{(b t / x)^{1 / \alpha}} z^{n-1} d z,
\end{aligned}
$$

but the last integral is trivially evaluated, and for the $n$th cumulant we have

$$
\left\langle\left\langle X^{n}(t)\right\rangle\right\rangle=\frac{i^{-n} \widetilde{h}^{(n)}(0)}{n}(b t)^{n / \alpha} \int_{0}^{\infty} \frac{\phi^{n}(x)}{x^{n / \alpha}} d x .
$$

Taking into account that $\widetilde{h}^{(n)}(0)=0$ for $n$ odd [we have assumed a symmetric jump distribution $h(x)$ ], we write

$$
\left\langle\left\langle X^{2 n-1}(t)\right\rangle\right\rangle=0
$$

and 


$$
\left\langle\left\langle X^{2 n}(t)\right\rangle\right\rangle=\frac{(-1)^{n} \widetilde{h}^{(2 n)}(0)}{2 n}(b t)^{2 n / \alpha} \int_{0}^{\infty} \frac{\phi^{2 n}(x)}{x^{2 n / \alpha}} d x
$$

$(n=1,2,3, \ldots)$. In Appendix $\mathrm{C}$ we check the convergence of these expressions and hence show the existence of moments and cumulants. Specifically, we prove that if the pulse function has the asymptotic behaviors

$$
\phi(x) \sim x^{\beta} \quad(x \rightarrow 0),
$$

$(\beta>0)$ and

$$
\phi(x) \sim x^{\gamma} \quad(x \rightarrow \infty),
$$

then all cumulants will exist if

$$
\frac{1}{\gamma+1 / 2}>\alpha>\frac{1}{\beta}
$$

Note that for a steplike function, such as that of Eq. (33), where $\gamma=0$ all cumulants will exist if

$$
2>\alpha>1 / \beta .
$$

Finally, for any integrable function $\phi(t)$ over $[0, \infty)$ there is no upper bound for $\alpha$ and the only condition on $\alpha$ for having all moments finite is that $\alpha>1 / \beta$.

We close this discussion on moments and cumulants with an example. Suppose that the pulse function is given by the steplike function (33). In this case $\gamma=0, \beta=1$ and all moments (and cumulants) will exist if $1<\alpha<2$. Cumulants are given by Eqs. (36) and (37). In Appendix B we show that

$$
\int_{0}^{\infty} \frac{\left(1-e^{-k x}\right)^{n}}{x^{n}} d x=A_{n} k^{-1+n / \alpha} \Gamma(1+n-n / \alpha),
$$

where the numbers $A_{n}$ are given by Eq. (B3) of Appendix B. Finally,

$$
\begin{aligned}
\left\langle\left\langle X^{2 n}(t)\right\rangle\right\rangle= & \frac{(-1)^{n} \widetilde{h}^{(2 n)}(0)}{2 n} A_{2 n} k^{-1+2 n / \alpha} \\
& \times \Gamma(1+2 n-2 n / \alpha)(b t)^{2 n / \alpha} .
\end{aligned}
$$

We finish this section by evaluating the power spectrum of the integrated process $X(t)$. Let us first evaluate the correlation function

$$
\langle X(t+\tau) X(t)\rangle=-\left.\frac{\partial^{2}}{\partial \omega_{1} \omega_{2}} \tilde{p}\left(\omega_{1}, t ; \omega_{2}, t+\tau\right)\right|_{\omega_{1}=\omega_{2}=0} .
$$

From Eq. (28) we get

$$
\langle X(t+\tau) X(t)\rangle=b \int_{0}^{\infty} \frac{d z}{z^{1+\alpha}} \int_{0}^{t} \phi\left(b z^{-\alpha} t^{\prime}\right) \phi\left(b z^{-\alpha}\left(t^{\prime}+\tau\right)\right) .
$$

Let $C(\tau)$ be the correlation function in the stationary limit $t \rightarrow \infty$, i.e.,

$$
C(\tau)=\lim _{t \rightarrow \infty}\langle X(t+\tau) X(t)\rangle
$$

From Eq. (43) we have

$$
C(\tau)=b \int_{0}^{\infty} \frac{d z}{z^{1+\alpha}} \int_{0}^{\infty} \phi\left(b z^{-\alpha} t^{\prime}\right) \phi\left(b z^{-\alpha}\left(t^{\prime}+\tau\right)\right) .
$$

Note that the (stationary) variance $C(0)=\infty$, which agrees with the superdiffusive behavior of $X(t)$ given by Eq. (34).

The power spectral density of our process is thus given by the Fourier transform of the stationary correlation function

$$
\widetilde{C}(\omega)=\int_{-\infty}^{\infty} e^{-i \omega \tau} C(\tau) d \tau .
$$

Substituting Eq. (44) into this equation, performing simple changes of variables, and taking into account the causality of the pulse function $\phi(t)$, we finally obtain

$$
\widetilde{C}(\omega)=\frac{K}{\omega^{1+2 / \alpha}},
$$

where

$$
K=\frac{b^{2 / \alpha}}{\alpha} \int_{0}^{\infty} \xi^{2 / \alpha}|\widetilde{\phi}(\xi)|^{2} d \xi
$$

and $\widetilde{\phi}(\xi)$ is the Fourier transform of $\phi(t)$. We thus see that $X(t)$ has a power-law spectrum with exponent $\nu=1+2 \alpha$. In Appendix $\mathrm{C}$ we show that this exponent is bounded by

$$
1<\nu<2(1+\beta),
$$

where $\beta$ is defined in Eq. (38). Moreover, when $\phi(x)$ is analytic at $x=0$ then $\beta=1,2,3, \ldots$ is a positive integer and $X(t)$ is flicker noise $1 / f^{\nu}$ with $1<\nu<2(1+n) \quad(n$ $=1,2,3, \ldots)$. We finally observe that a power-law spectrum such as Eq. (45) exists if and only if the pulse shape function $\phi(t)$ possesses a Fourier transform. Note that this is not the case for the step function (29) or any steplike function such as (33).

\section{ASYMPTOTIC BEHAVIOR}

We will now examine the asymptotic behavior of the onetime probability density function of the integrated process $X(t), p(x, t) d x=\operatorname{Prob}\{x<X(t)<x+d x\}$. For this analysis we distinguish two regions: the "center" $(x \rightarrow 0)$ and the "tails" $(x \rightarrow \pm \infty)$ of the distribution. We cannot have a closed expression for the PDF $p(x, t)$ until the pulse function $\phi(x)$ and the jump PDF $h(x)$ are both specified. Therefore, we will perform the asymptotic on the $\mathrm{CF} \tilde{p}(\omega, t)$. As a well known feature of the harmonic analysis the center of the distribution is determined by the large $\omega$ behavior of the CF, while the tails are determined by $\tilde{p}(\omega, t)$ when $\omega \rightarrow 0$ [24].

We deal first with the center of the distribution where $\omega$ $\rightarrow \infty$. If we assume that the pulse function $\phi(x)$, as $x \rightarrow \infty$, satisfies Eq. (39), 


$$
\phi(x) \sim x^{\gamma} \quad(x \rightarrow \infty)
$$

then

$$
\widetilde{h}\left(z \phi\left(b t \omega^{\alpha} s / z^{\alpha}\right)\right) \sim \widetilde{h}\left(\left(b t \omega^{\alpha}\right)^{\gamma} z^{1-\alpha \gamma} s^{\gamma}\right) \quad(\omega \rightarrow \infty) .
$$

Substituting this into Eq. (25) and performing the change of variables $\xi=\left(b t \omega^{\alpha}\right)^{\gamma} z^{1-\alpha \gamma}$ we obtain the following Lévy distribution:

$$
\tilde{p}(\omega, t) \simeq \exp \left\{-L(b t)^{1 /(1-\alpha \gamma)} \omega^{\alpha /(1-\alpha \gamma)}\right\} \quad(\omega \rightarrow \infty),
$$

where

$$
L=\frac{1}{1-\alpha \gamma} \int_{0}^{\infty} \frac{d \xi}{\xi^{(1+\alpha-\alpha \gamma) /(1-\alpha \gamma)}}\left[1-\int_{0}^{1} \tilde{h}\left(\xi s^{\gamma}\right) d s\right]
$$

Note that due to the bounds discussed above [see Eq. (C2)] we have $1-\alpha \gamma>\alpha / 2$; hence the Lévy exponent in Eq. (48) satisfies

$$
0<\frac{\alpha}{1-\alpha \gamma}<2
$$

and Eq. (48) is well defined. We also note that for a steplike pulse function $\phi(t)$ where $\gamma=0$ we obtain the same Lévy distribution, Eq. (30), that satisfies the model for sudden pulses (29). Therefore, for any pulse shape function satisfying condition (39) the center of the PDF is given by a Lévy distribution.

Let us now obtain an asymptotic expression of the PDF $p(x, t)$ when $x \rightarrow \pm \infty$, which will be valid if $\phi(t)$ obeys Eqs. (38) and (39), and the exponent $\alpha$ is bounded by

$$
\beta^{-1}<\alpha<1 /(\gamma+1 / 2)
$$

In this case, we see from Eq. (40) that all cumulants exist. So taking the $\omega \rightarrow 0$ limit of Eq. (26) we find

$$
\tilde{p}(\omega, t) \sim 1-b t \int_{0}^{\infty} \frac{d z}{z^{1+\alpha}}\left[1-\int_{0}^{1} \tilde{h}\left(z \omega \phi\left(b t s / z^{\alpha}\right)\right) d z\right] .
$$

The Fourier inversion of Eq. (51) yields

$$
p(x, t) \sim b t \int_{0}^{\infty} \frac{d z}{z^{1+\alpha}} \int_{0}^{1} h(x, z, s) d z \quad(x \rightarrow \pm \infty),
$$

where we have dropped $\delta$ function terms that have no contribution as $x \rightarrow \infty$. Moreover, assuming symmetric jump distributions $h(x)$ we have

$$
\begin{aligned}
h(x, z, s) & \equiv \frac{1}{\pi} \int_{0}^{\infty} \tilde{h}\left(z \omega \phi\left(b t s / z^{\alpha}\right)\right) \cos \omega x d \omega \\
& =\frac{1}{z \phi\left(b t s / z^{\alpha}\right)} h\left(\frac{x}{z \phi\left(b t s / z^{\alpha}\right)}\right) .
\end{aligned}
$$

Substituting this into Eq. (52) we see that

$$
\begin{gathered}
p(x, t) \sim b t \int_{0}^{\infty} \frac{d z}{z^{2+\alpha}} \int_{0}^{1} \frac{d s}{\phi\left(b t s / z^{\alpha}\right)} h\left(\frac{x}{z \phi\left(b t s / z^{\alpha}\right)}\right) \\
(x \rightarrow \pm \infty) .
\end{gathered}
$$

Therefore the tails of the distribution are determined by the jump PDF $h(x)$ and the pulse shape function $\phi(t)$.

Finally, for the rectangular pulse (29) we have

$$
p(x, t) \sim \frac{b t}{|x|^{1+\alpha}} \int_{0}^{\infty} y^{\alpha} h(y) d y,
$$

which agrees with the expected tail behavior of the Lévy distribution [8].

\section{RELATION TO THE LÉVY DISTRIBUTION}

In Sec. V we obtained general expressions (36) and (37) for all the cumulants, from which it follows that

$$
\begin{aligned}
\ln \tilde{p}(\omega, t) & =\sum_{n=1}^{\infty} \frac{i^{n} \omega^{n}}{n !}\left\langle\left\langle X^{n}(t)\right\rangle\right\rangle \\
& =\sum_{n=1}^{\infty} \frac{(-1)^{n} \omega^{2 n} \widetilde{h}^{(2 n)}(0)}{2 n(2 n) !}(b t)^{2 n / \alpha} \int_{0}^{1} \frac{\phi^{2 n}(x)}{x^{2 n / \alpha}} d x .
\end{aligned}
$$

In addition, in Sec. VI, we showed that $p(x, t)$ was a Lévy distribution at the center of the distribution $(x \rightarrow 0)$ and took the form (53) in the tails $(x \rightarrow \pm \infty)$ of the distribution. In this section we will show how the distribution can be separated into a Lévy distribution plus an additional term. This term takes the form of a single integral which can be evaluated once the functions $\phi$ and $h$ have been specified.

We begin the analysis by changing variables from $s$ to $x$ $=b t s / z^{\alpha}$ ( $z$ fixed) in Eq. (26). This gives

$$
\begin{aligned}
\ln \tilde{p}(\omega, t) & =-\int_{0}^{\infty} \frac{d z}{z} \int_{0}^{b t / z^{\alpha}} d x\{1-\widetilde{h}(z \omega \phi(x))\} \\
& =-\int_{0}^{\infty} d x \int_{0}^{(b t / x)^{1 / \alpha}} \frac{d z}{z}\{1-\tilde{h}(z \omega \phi(x))\},
\end{aligned}
$$

changing the order of integration. At this point we factor out the contribution from the Lévy process by writing Eq. (56) as

$$
\begin{aligned}
& -\int_{0}^{\infty} d x \int_{0}^{(b t / x)^{1 / \alpha}} \frac{d z}{z}\{1-\tilde{h}(z \omega)\} \\
& -\int_{0}^{\infty} d x \int_{0}^{(b t / x)^{1 / \alpha}} \frac{d z}{z}\{\tilde{h}(z \omega)-\tilde{h}(z \omega \phi(x))\},
\end{aligned}
$$

or, after defining

$$
\widetilde{g}(\omega, t) \equiv 1-\widetilde{h}(\omega, t),
$$


as

$$
\begin{aligned}
& -\int_{0}^{\infty} d x \int_{0}^{(b t / x)^{1 / \alpha}} \frac{\widetilde{g}(\omega, t)}{z} d z \\
& \quad+\int_{0}^{\infty} d x \int_{0}^{(b t / x)^{1 / \alpha}} \frac{d z}{z}\{\tilde{g}(z \omega)-\tilde{g}(z \omega \phi(x))\} .
\end{aligned}
$$

The first term is just $\ln \tilde{p}(\omega, t)$ for Lévy processes [see Eqs. (30) and (31)]. The second term can be simplified by first writing it as

$$
\int_{0}^{\infty} d x \int_{(b t / x)^{1 / \alpha} \phi}^{(b t / x)^{1 / \alpha}} \frac{\tilde{g}(z \omega)}{z} d z
$$

and then integrating by parts to give

$$
\left|x \int_{(b t / x)^{1 / \alpha} \phi}^{(b t / x)^{1 / \alpha}} \frac{\tilde{g}(z \omega)}{z} d z\right|_{x=0}^{x=\infty}-\int_{0}^{\infty} d x x \frac{\partial}{\partial x} \int_{(b t / x)^{1 / \alpha} \phi}^{(b t / x)^{1 / \alpha}} \frac{\tilde{g}(z \omega)}{z} d z .
$$

We assume that $\tilde{h}(\omega)$ is analytic at $\omega=0$ and integrable; then

$$
\tilde{g}(\omega) \sim \omega^{2} \quad(\omega \rightarrow 0) \quad \text { and } \quad \tilde{g}(\omega) \rightarrow 1 \quad(\omega \rightarrow \infty),
$$

and since $0<\alpha<2$ the first term in Eq. (58) is zero. Finally,

$$
\begin{aligned}
\ln \tilde{p}(\omega, t)= & \ln \tilde{p}_{\text {Lévy }}(\omega, t)+\frac{1}{\alpha} \int_{0}^{\infty}\left[\tilde{g}\left([b t / x]^{1 / \alpha} \omega\right)\right. \\
& \left.-\tilde{g}\left([b t / x]^{1 / \alpha} \omega \phi(x)\right)\right] d x \\
& +\int_{0}^{\infty} x \frac{\phi^{\prime}(x)}{\phi(x)} \tilde{g}\left([b t / x]^{1 / \alpha} \omega \phi(x)\right) d x
\end{aligned}
$$

where $\phi^{\prime}(x)$ is the derivative of the pulse shape function and

$$
\ln \tilde{p}_{\text {Lévy }}(\omega, t)=-M t \omega^{\alpha},
$$

where $M$ is given by Eq. (31). Note that when $\phi(x)$ is the Heaviside step function the integrals on the right hand side of Eq. (59) vanish and Eq. (59) reduces to the Lévy distribution. Therefore, we can look at the second term on the right hand side of Eq. (59) as a correction to the Lévy distribution when $\phi(x)$ is not exactly a Heaviside function but a steplike function very close to the Heaviside function. This may be evaluated, in principle, for any given $\phi$ and $\tilde{h}$. For instance, we could take $\phi$ to be of the form (33) with $k$ large and the Lorentzian

$$
\tilde{g}(\omega)=\frac{\omega^{2} / 2}{1+\omega^{2} / 2}
$$

corresponding to $h(x)=e^{-\sqrt{2}|x|} / \sqrt{2}$. The form of the correction terms depends on the choice of the functions to an extent, and so we will not discuss the explicit form it takes here.

\section{CONCLUSIONS}

In this paper we have presented and analyzed a dynamical model based on a process that is a superposition of colored Poisson noises. The model was shown to have several attractive features. The probability density function has long tails, which emerged in a natural way and, unlike the Lévy distribution, all the moments of the distribution are finite. We believe that these properties make the distribution an ideal candidate for describing stock market prices [6].

Another property that may have relevance to physics and other natural sciences is the appearance of a power-law spectrum for the process. Once again we would like to stress that this result flowed naturally from the nature of the model and the scaling assumptions, which reduce $h$ and $\phi$ from functions of two variables to functions of a single variable.

In a more mathematical context, we believe that the decomposition of the CF of our model into that of the Lévy plus additional terms is interesting, both as an example of an Edgeworth-type expansion and for the nature of the corrections to the Lévy distributions when the parameters of our model are chosen so that our distribution is near to the Lévy one.

There are still some open questions. One of them is the extension of the model to the increments of the process $Z\left(\tau, t-t_{0}\right)=X\left(t-t_{0}+\tau\right)-X\left(t-t_{0}\right) \quad\left(t>t_{0}\right)$, since in this case we believe that the process $Z\left(\tau, t-t_{0}\right)$ becomes stationary when it starts in the infinite past $\left(t_{0} \rightarrow-\infty\right)$. Another interesting and open question is the actual application of the model to financial time series where some nonwhite correlation is observed [25]. Both points are presently being investigated.

\section{ACKNOWLEDGMENTS}

This work was supported in part by Dirección General de Investigación under Contract No. BFM2000-0795 and Project No. HB119-0104, and by Generalitat de Catalunya under Contract No. 2000 SGR-00023 (J.M. and M.M.). A.M. thanks the British Council for support under the "Acciones Integradas"' scheme.

\section{APPENDIX A: CHARACTERISTIC FUNCTION FOR COLORED SHOT NOISE}

By generalizing Rice's method [20], we will now obtain the probability distribution of the shot noise $Y(u, t)$ defined by Eq. (2):

$$
Y(u, t)=\sum_{k=1}^{\infty} A_{k}(u) \phi\left(t-T_{k}(u) ; u\right)
$$

where we assume that the random variables $A_{k}(u)$ and $T_{k}(u)$ are identically distributed and statistically independent. The jump amplitudes are described by the PDF $h(x, u) d x$ $=\operatorname{Prob}\left\{x<A_{k}(u)<x+d x\right\}$ and the jump times $T_{k}(u)$ follow a Poisson distribution of the parameter $\lambda(u)$. Define 


$$
\begin{aligned}
& p\left(x_{1}, t_{1} ; x_{2}, t_{2} ; u\right) d x_{1} d x_{2} \\
& \quad=\operatorname{Prob}\left\{x_{1}<Y\left(u, t_{1}\right)<x_{1}+d x_{1} ; x_{2}<Y\left(u, t_{2}\right)<x_{2}+d x_{2}\right\}
\end{aligned}
$$

to be the joint PDF of the process with $t_{2} \geqslant t_{1}$. This PDF can be written as

$$
\begin{aligned}
& p\left(x_{1}, t_{1} ; x_{2}, t_{2} ; u\right) \\
& \quad=\sum_{n_{1}=0}^{\infty} \sum_{n_{2}=0}^{\infty} p\left(x_{1}, t_{1} ; x_{2}, t_{2} ; u \mid n_{1}, n_{2}\right) P\left(n_{1}, t_{1} ; n_{2}, t_{2} ; u\right),
\end{aligned}
$$

where $p\left(x_{1}, t_{1} ; x_{2}, t_{2} ; u \mid n_{1}, n_{2}\right)$ is the conditional PDF assuming that exactly $n_{1}$ pulses have occurred at time $t_{1}$ and $n_{2}$ pulses at time $t_{2} . P\left(n_{1}, t_{1} ; n_{2}, t_{2} ; u\right)$ is the joint probability for the occurrence of such pulses. Since $t_{2} \geqslant t_{1}$ then $n_{2}$ $\geqslant n_{1}$ and

$$
\begin{aligned}
& P\left(n_{1}, t_{1} ; n_{2}, t_{2} ; u\right) \\
& \quad= \begin{cases}P\left(n_{2}-n_{1} ; t_{2}-t_{1} ; u\right) P\left(n_{1}, t_{1} ; u\right) & \text { if } n_{1} \leqslant n_{2} \\
0 & \text { otherwise, }\end{cases}
\end{aligned}
$$

where

$$
P(m, \tau ; u)=\frac{[\lambda(u) \tau]^{m}}{m !} e^{-\lambda(u) \tau}
$$

is the Poisson distribution. Substituting Eq. (A3) into Eq. (A2) and defining $t_{1}=t, t_{2}=t+\Delta t, n_{1}=n$, and $n_{2}-n_{1}=m$, we obtain

$$
\begin{aligned}
p\left(x_{1}, t ; x_{2}, t+\Delta t ; u\right)= & \sum_{n=0}^{\infty} \sum_{m=0}^{\infty} p\left(x_{1}, t ; x_{2}, t+\Delta t ; u \mid n, n\right. \\
& +m) P(m, \Delta t ; u) P(n, t ; u),
\end{aligned}
$$

and the characteristic function reads

$$
\begin{aligned}
\tilde{p}\left(\omega_{1}, t ; \omega_{2}, t+\Delta t ; u\right)= & \sum_{n=0}^{\infty} \sum_{m=0}^{\infty} \tilde{p}\left(\omega_{1}, t ; \omega_{2}, t+\Delta t ; u \mid n, n\right. \\
& +m) P(m, \Delta t ; u) P(n, t ; u) .
\end{aligned}
$$

Note that $\tilde{p}\left(\omega_{1}, t ; \omega_{2}, t+\Delta t ; u \mid n, n+m\right)$ is the joint characteristic function of the truncated process

$$
Y_{n}(u, t)=\sum_{k=1}^{n} A_{k}(u) \phi\left(t-T_{k}(u) ; u\right) .
$$

$$
\begin{aligned}
\tilde{p}\left(\omega_{1}, t ; \omega_{2}, t+\Delta t ; u \mid n, n+m\right) \\
=\left\langle\operatorname { e x p } \left[ i \omega_{2} \sum_{j=1}^{n} A_{j}(u) \phi\left(t+\Delta t-T_{j}(u) ; u\right)\right.\right. \\
\quad+i \omega_{1} \sum_{k=1}^{n} A_{k}(u) \phi\left(t-T_{k}(u) ; u\right) \\
\left.\left.\quad+i \omega_{2} \sum_{l=n+1}^{n+m} A_{l}(u) \phi\left(t+\Delta t-T_{l}(u) ; u\right)\right]\right\rangle .
\end{aligned}
$$

Taking into account that $A_{k}(u)$ and $T_{k}(u)$ are independent and identically distributed random variables, we have

$$
\begin{aligned}
\tilde{p}\left(\omega_{1}, t\right. & \left.; \omega_{2}, t+\Delta t ; u \mid n, n+m\right) \\
= & {\left[\left\langle\operatorname { e x p } \left\{i \omega_{2} A_{j}(u) \phi\left(t+\Delta t-T_{j}(u) ; u\right)\right.\right.\right.} \\
& \left.\left.\left.+i \omega_{1} A_{k}(u) \phi\left(t-T_{k}(u) ; u\right)\right\}\right\rangle\right]^{n} \\
& \times\left[\left\langle\exp \left\{i \omega_{2} A_{l}(u) \phi\left(t+\Delta t-T_{l}(u) ; u\right)\right\}\right\rangle\right]^{m},
\end{aligned}
$$

and, since the random times $T_{k}(u)$ are Poissonian,

$$
\begin{aligned}
& \left\langle\operatorname { e x p } \left\{ i \omega_{2} A_{k}(u) \phi\left(t+\Delta t-T_{k}(u) ; u\right)\right.\right. \\
& \left.\left.+i \omega_{1} A_{k}(u) \phi\left(t-T_{k}(u) ; u\right)\right\}\right\rangle \\
& =\int_{-\infty}^{\infty} h(a, u) d a \int_{0}^{t} \frac{d t^{\prime}}{t} \exp \left\{i a \left[\omega_{2} \phi\left(t+\Delta t-t^{\prime} ; u\right)\right.\right. \\
& \left.\left.\quad+\omega_{1} \phi\left(t-t^{\prime} ; u\right)\right]\right\}=\frac{1}{t} \int_{0}^{t} \tilde{h}\left(\omega_{2} \phi\left(t+\Delta t-t^{\prime} ; u\right)\right. \\
& \left.\quad+\omega_{1} \phi\left(t-t^{\prime} ; u\right) ; u\right) d t^{\prime},
\end{aligned}
$$

where $\widetilde{h}(\omega ; u)$ is the Fourier transform of the jump PDF $h(x ; u)$. Analogously,

$$
\begin{aligned}
& \left\langle\exp \left\{i \omega_{2} A_{k}(u) \phi\left(t+\Delta t-T_{k}(u) ; u\right)\right\}\right\rangle \\
& =\frac{1}{\Delta t} \int_{t}^{t+\Delta t} \tilde{h}\left(\omega_{2} \phi\left(t+\Delta t-t^{\prime} ; u\right) ; u\right) d t^{\prime} .
\end{aligned}
$$

Therefore,

$$
\begin{aligned}
& \tilde{p}\left(\omega_{1}, t ; \omega_{2}, t+\Delta t ; u \mid n, n+m\right) \\
& \quad=\left[\frac{1}{t} F\left(\omega_{2}, t+\Delta t ; \omega_{1}, t ; u\right)\right]^{n}\left[\frac{1}{\Delta t} G\left(\omega_{2}, t+\Delta t ; t ; u\right)\right]^{m},
\end{aligned}
$$

where

$$
\begin{aligned}
& F\left(\omega_{2}, t+\Delta t ; \omega_{1}, t ; u\right) \\
& \quad \equiv \int_{0}^{t} \widetilde{h}\left(\omega_{2} \phi\left(t+\Delta t-t^{\prime} ; u\right)+\omega_{1} \phi\left(t-t^{\prime} ; u\right) ; u\right) d t^{\prime}
\end{aligned}
$$

and 


$$
G\left(\omega_{2}, t+\Delta t ; t ; u\right) \equiv \int_{t}^{t+\Delta t} \widetilde{h}\left(\omega_{2} \phi\left(t+\Delta t-t^{\prime} ; u\right) ; u\right) d t^{\prime} .
$$

Substituting Eq. (A6) into Eq. (A5) yields

$$
\begin{aligned}
\tilde{p}\left(\omega_{1}, t ; \omega_{2}, t+\Delta t ; u\right) & \\
= & \left\{\sum_{n=0}^{\infty}\left[\frac{1}{t} F\left(\omega_{1}, t ; \omega_{2}, t+\Delta t ; u\right)\right]^{n} P(n, t ; u)\right\} \\
& \times\left\{\sum_{m=0}^{\infty}\left[\frac{1}{\Delta t} G\left(\omega_{2}, t+\Delta t ; t ; u\right)\right]^{m} P(m, \Delta t ; u)\right\} .
\end{aligned}
$$

Introducing Eq. (A4) into this and performing the resulting sums we get

$$
\begin{aligned}
\tilde{p}\left(\omega_{1}, t ; \omega_{2}, t+\Delta t ; u\right) & \\
= & \exp \left\{-\lambda(u)\left[t-F\left(\omega_{2}, t+\Delta t ; \omega_{1}, t ; u\right)\right]\right. \\
& \left.-\lambda(u)\left[\Delta t-G\left(\omega_{2}, t+\Delta t ; \omega_{1}, t ; u\right)\right]\right\} .
\end{aligned}
$$

Finally,

$$
\begin{aligned}
\tilde{p}\left(\omega_{1}, t ; \omega_{2}, t+\Delta t ; u\right) & \\
= & \exp \left\{\lambda ( u ) \int _ { 0 } ^ { t } d t ^ { \prime } \left[\widetilde{h}\left(\omega_{1} \phi\left(t^{\prime}, u\right)+\omega_{2} \phi\left(t^{\prime}+\Delta t, u\right)\right)\right.\right. \\
& \left.-1]+\lambda(u) \int_{0}^{\Delta t} d t^{\prime}\left[\widetilde{h}\left(\omega_{2} \phi\left(t^{\prime}, u\right)\right)-1\right]\right\}, \quad \text { (A9) }
\end{aligned}
$$

which agrees with Eq. (7). If in Eq. (A9) we set $\omega_{1}=\omega$ and $\omega_{2}=0$, we obtain the one-time characteristic function (6):

$$
\tilde{p}(\omega, t ; u)=\exp \left\{\lambda(u) \int_{0}^{t} d t^{\prime}\left[\tilde{h}\left(\omega \phi\left(t^{\prime}, u\right)\right)-1\right]\right\} .
$$

\section{APPENDIX B: CUMULANTS FOR A STEPLIKE FUNCTION}

We will derive closed expressions for the cumulants (37)

$$
\left\langle\left\langle X^{2 n}(t)\right\rangle\right\rangle=\frac{(-1)^{n} \widetilde{h}^{(2 n)}(0)}{2 n}(b t)^{2 n / \alpha} \int_{0}^{\infty} \frac{\phi^{2 n}(x)}{x^{2 n / \alpha}} d x,
$$

when $\phi(x)$ is the steplike function (33)

$$
\phi(t)= \begin{cases}1-e^{-k t} & \text { if } t>0 \\ 0 & \text { otherwise }\end{cases}
$$

The substitution of Eq. (B2) into Eq. (B1) leads us to evaluate the following integral:

$$
\begin{aligned}
I_{2 n}= & \int_{0}^{\infty} \frac{\left(1-e^{-k x}\right)^{2 n}}{x^{2 n / \alpha}} d x=\int_{0}^{\infty} \frac{d x}{x^{2 n / \alpha}}\left[k x \int_{0}^{1} e^{-k x u} d u\right]^{2 n} \\
= & k^{2 n} \int_{0}^{1} d u_{1} \cdots \int_{0}^{1} d u_{2 n} \int_{0}^{\infty} x^{2 n(1-1 / \alpha)} \\
& \times \exp \left\{-k x\left(\sum_{i=1}^{2 n} u_{i}\right)\right\} d x .
\end{aligned}
$$

Define the new integration variable

$$
\xi=k x\left(\sum_{i=1}^{2 n} u_{i}\right)
$$

then

$$
\begin{aligned}
I_{2 n}= & k^{-1+2 n / \alpha} \int_{0}^{1} d u_{1} \cdots \int_{0}^{1} d u_{2 n}\left(\sum_{i=1}^{2 n} u_{i}\right)^{-1-2 n(1-1 / \alpha)} \\
& \times \int_{0}^{\infty} e^{-\xi} \xi^{2 n(1-1 / \alpha)} d \xi
\end{aligned}
$$

But

$$
\int_{0}^{\infty} e^{-\xi} \xi^{2 n(1-1 / \alpha)} d \xi=\Gamma(1+2 n-2 n / \alpha) .
$$

Defining the numbers

$$
A_{2 n} \equiv \int_{0}^{1} d u_{1} \cdots \int_{0}^{1} d u_{2 n}\left(\sum_{i=1}^{2 n} u_{i}\right)^{-1-2 n(1-1 / \alpha)}
$$

we finally have

$$
\begin{aligned}
\left\langle\left\langle X^{2 n}(t)\right\rangle\right\rangle= & \frac{(-1)^{-n} \widetilde{h}^{(2 n)}(0)}{2 n} A_{2 n} k^{-1+2 n / \alpha} \\
& \times \Gamma(1+2 n-2 n / \alpha)(b t)^{2 n / \alpha},
\end{aligned}
$$

which is Eq. (42). Note that when $n=1$ Eq. (B4) reduces to Eq. (34).

\section{APPENDIX C: CONVERGENCE OF INTEGRALS}

Let us first check the existence of cumulants (37). Assuming the asymptotic behavior given by Eq. (38), then the convergence of the integral on the right hand side of Eq. (37) as $x \rightarrow 0$ implies that the scaling exponent $\alpha$ has a lower bound:

$$
\alpha>\frac{2 n}{1+2 n \beta} .
$$

On the other hand, assuming Eq. (39) then the convergence of Eq. (37) when $x \rightarrow \infty$ implies that the scaling exponent $\alpha$ also has an upper bound:

$$
\frac{1}{\alpha}>\gamma+\frac{1}{2 n} .
$$


Moreover, when $\gamma \geqslant 0$ then if

$$
\frac{1}{\gamma+1 / 2}>\alpha>\frac{1}{\beta}
$$

all cumulants will exist. Note that Eq. (C2) holds whenever $\gamma \geqslant-1 / 2 n$. Therefore, $\gamma \geqslant 0$ is a sufficient condition for its validity. Finally, if $\gamma<-1 / 2$ there is no upper bound on the accepted values of $\alpha$.

We now prove the existence of the power spectral density $\widetilde{C}(\omega)$ given by Eq. (45). Obviously this density will exist if the integral

$$
J=\int_{0}^{\infty} \xi^{2 / \alpha}|\widetilde{\phi}(\xi)|^{2} d \xi<\infty .
$$

In order to prove the existence of $J$ we first need to show that the Fourier transform of the pulse function, $\widetilde{\phi}(\xi)$, exists. Note that any step or steplike function does not have a Fourier transform and consequently the power spectrum is infi- nite. For the existence of $\widetilde{\phi}(\xi)$ it suffices that $\phi(x)$ be absolutely integrable, and from the asymptotic behavior given by Eq. (39) we have to impose that $\gamma<-1$. This in turn implies that $\widetilde{\phi}(\xi) \sim \xi^{-1-\gamma}$ as $\xi \rightarrow 0$ and, since $\gamma<-1$, the integral $J$ at its lower limit is always finite for any $\alpha>0$. On the other hand, if $\phi(x)$ satisfies Eq. (38) as $x \rightarrow 0$, then $\widetilde{\phi}(\xi)$ $\sim \xi^{-1-\beta}$ as $\xi \rightarrow \infty$. Hence, $J$ will be finite if $\alpha>1 /(\beta+1 / 2)$ [see also Eq. $(\mathrm{C} 1)]$. Therefore, the process $X(t)$ has a finite power spectrum if $\phi(x)$ is absolutely integrable on the real line, and $X(t)$ has a finite second cumulant Eq. (C1), i.e.,

$$
\alpha>\frac{1}{\beta+1 / 2} .
$$

In such a case, we see from Eq. (45) that $X(t)$ exhibits a power-law spectrum with exponent $\nu=1+2 / \alpha$. Moreover [recall that $\alpha>0$, and Eq. (C4)],

$$
1<\nu<2(1+\beta)
$$

[1] The Random Character of Stock Market Prices, edited by P. H. Cootner (MIT Press, Cambridge, MA, 1964).

[2] Apparently Kendall was the first to notice that a normal distribution did not fit financial data, especially at the wings of the distribution: M. G. Kendall, J. R. Stat. Soc. 96, 11 (1953).

[3] B. Mandelbrot, J. Business 35, 394 (1963); E. Fama, ibid. 35, 420 (1963).

[4] R. N. Mantegna and H. E. Stanley, Phys. Rev. Lett. 73, 2946 (1994); I. Koponen, Phys. Rev. E 52, 1197 (1995).

[5] R. N. Mantegna and H. E. Stanley, Nature (London) 376, 46 (1995); An Introduction to Econophysics (Cambridge University Press, Cambridge, 2000).

[6] J. Masoliver, M. Montero, and J. M. Porrá, Physica A 283, 559 (2000).

[7] H. Cramer, Mathematical Methods of Statistics (Princeton University Press, Princeton, NJ, 1951).

[8] Lévy Flights and Related Topics in Physics, edited by M. F. Shlesinger, G. M. Zaslavsky, and U. Frisch, Lecture Notes in Physics Vol. 450 (Springer-Verlag, Berlin, 1995).

[9] E. Barkai, R. Silbey, and G. Zumofen, Phys. Rev. Lett. 84, 5339 (2000).

[10] F. Bardou, J. P. Bouchaud, O. Emile, A. Aspect, and C. Cohen-Tannoudji, Phys. Rev. Lett. 72, 203 (1994).

[11] B. Saubaméa, M. Leduc, and C. Cohen-Tannoudji, Phys. Rev. Lett. 83, 3796 (1999).

[12] S. Marksteiner, K. Ellinger, and P. Zoller, Phys. Rev. A 53, 3409 (1996); W. Greenwood, P. Pax, and P. Meystre, ibid. 56,
2109 (1997).

[13] H. Katori, S. Schlipf, and H. Walther, Phys. Rev. Lett. 79, 2221 (1997).

[14] J. Reichel, F. Bardou, M. Ben Dahan, E. Peik, S. Rand, C. Salomon, and C. Cohen-Tannoudji, Phys. Rev. Lett. 75, 4575 (1995).

[15] A. Ott, J. P. Bouchaud, D. Langevin, and W. Urbach, Phys. Rev. Lett. 65, 2201 (1990).

[16] S. C. Venkataramani, T. M. Antonsen, and E. Ott, Phys. Rev. Lett. 78, 3864 (1997).

[17] D. Bénisti and D. F. Escande, Phys. Rev. Lett. 80, 4871 (1998).

[18] V. Latora, A. Rapisarda, and S. Ruffo, Phys. Rev. Lett. 83, 2104 (1999).

[19] E. Lukacs, Characteristic Functions (Griffin, London, 1970).

[20] S. O. Rice, in Noise and Stochastic Processes, edited by N. Wax (Dover, New York, 1954).

[21] S. M. Rytov, Yu. A. Kravtsov, and V. I. Tatarskii, Principles of Statistical Radiophysics (Springer-Verlag, Berlin, 1987).

[22] S. B. Lowen and M. C. Teich, Phys. Rev. Lett. 63, 1755 (1989).

[23] J. Masoliver, Phys. Rev. A 35, 3918 (1987).

[24] G. H. Weiss, Aspects and Applications of the Random Walk (North-Holland, Amsterdam, 1994).

[25] J. Y. Campbell, A. W. Lo, and A. C. MacKinlay, The Econometrics of Financial Markets (Princeton University Press, Princeton, NJ, 1997). 